\title{
Predictive genetic testing, risk communication, and risk perception: an international expert meeting in Berlin, Germany
}

\author{
Eva Fisher • Steffi Achilles • Holger Tönnies
}

Received: 21 November 2013 / Accepted: 22 November 2013 /Published online: 10 December 2013

(C) Springer-Verlag Berlin Heidelberg 2013

Use of the broad knowledge about human genetic variation for the benefit of human health gives rise to a huge range of challenges. One of these challenges was addressed at an international symposium held in Berlin in November 2011 entitled "Predictive Genetic Testing, Risk Communication and Risk Perception." A particular focus of this meeting was the question how patients or consumers deal with the knowledge about their own individual genetic risks, i.e., to what extent this knowledge might change their attitudes towards a healthy lifestyle and their consequent behavior, or whether, on the contrary, it creates psychological harm (anxiety or misconception, e.g., false reassurance), rather than benefit to their health. The scientific program was divided into three sections as follows: the first focused on the current status of risk prediction models for common diseases (breast cancer, type 2 diabetes) and the evidence-based evaluation of genetic tests, in particular, presymptomatic or probabilistic genetic tests for health care purposes; the second part was devoted to the communication of genetic risks to, and perception of genetic risks by healthy persons; the third part focused on the psychological and motivational impacts on persons who underwent genetic testing. The symposium was organized by the Administrative Office of the German Commission on Genetic Testing and financed by the German Federal Ministry of Health. In this special issue, some of the speakers present the thoughts and knowledge which they shared with the audience in November 2011 in Berlin. As a tribute to all speakers and for the convenience of the interested reader, this editorial provides brief summaries of the talks given at the symposium.

This article is part of the special issue "Predictive Genetic Testing, Risk Communication and Risk Perception."

E. Fisher $(\bowtie) \cdot S$. Achilles $\cdot H$. Tönnies

Administrative Office of the German Commission on Genetic

Testing, Robert Koch-Institute, Nordufer 20, 13353 Berlin, Germany

e-mail: FisherE@rki.de
The first talk was given by Douglas Easton (Center for Cancer Genetic Epidemiology, University of Cambridge, UK), who presented evidence for genetically predisposed subtypes of breast cancer, based on recent findings from genome-wide association studies. As Dr. Easton stated, most familial breast cancers are not due to high-risk genes like $B R C A 1$ and BRCA2. To date, 23 common loci are known, which, together with breast density measurements, attain a predictive power equal to that known from rare $B R C A$ mutations. Those known moderate risk variants are generally specific to clinical subtypes. Risk prediction based on common variants is, therefore, useful for high-risk individuals, but is not yet feasible in a wider application. Still, most causal variants are unknown. Since many different pathways are involved in breast cancer etiology and interaction multiplies those factors, genetic risk prediction has not reached such a stage that it is considered by physicians in the genetic counseling of high-risk families. Finally, Dr. Easton drew attention to the expected relevance of forthcoming results from ongoing efforts of large international consortia to identify rare variants by exome or genome sequencing.

Matthias Schulze (German Institute of Human Nutrition, Germany) discussed the current state of type 2 diabetes risk prediction models. He pointed out that models including all presently known common variants ( 40 SNPs) still have limited power to identify individuals in the general population at risk of developing diabetes with little improvement in precision compared to those models based solely on other commonly known risk factors (e.g., high BMI, lack of physical exercise, etc.). However, genetic risk prediction in younger persons $(<50$ years of age) showed higher potential to identify those who are at risk. Whether risk scores based on traditional and genetic risk factors may provide subgroup-specific evidence for early interventional strategies to delay disease onset in the healthy needs further validation. 
Dave Dotson (CDC's Office of Public Health Genomics (OPHG), USA) followed with his talk about the Evaluation of Genomic Applications in Practice and Prevention (EGAPP) Initiative, which was established in 2007 and serves as a longterm sustainable source of research translation into clinical practice. The EGAPP working group establishes recommendations for the applicability of genetic tests for medical purposes via systematic, evidence-based processes. The initiative pursues the principles of comprehensive transparency and publicity. Dr. Dotson introduced some of the working group's recent recommendations on genetic variants which have potential benefit for common disease prevention or which predict response to drug treatment. He also drew attention to GAPP Finder, launched by OPHG in 2010, which provides a continually updated database, tracking the growing number of genetic tests and genomic applications under development or available for use in clinical and public health practice.

Robert Green (Harvard-Partners Center for Personalized Genetic Medicine, USA) first gave some insights into the Risk Evaluation and Education for Alzheimer's Disease (REVEAL) study, in which adult offspring of Alzheimer's disease patients were offered testing for the apolipoprotein $\mathrm{E}$ (Apo E) polymorphism. At this point, Dr. Green addressed the major issue of the symposium - the perception and behavioral outcome of predictive genetic testing. The REVEAL study showed that testing had minimal psychological impact and even provoked behavioral changes (for example, intake of vitamins and other supplements or the taking out of new health insurances) in persons to whom the information that they were carriers of the high-risk Apo E 4-allele had been disclosed, although no effective preventive measures for Alzheimer's disease exist today. Dr. Green pointed out that, in the public and scientists' view, the road to "healthy aging" starts with self-awareness and self-responsibility towards disease prevention. To this end, action is needed early in life. However, solid scientific evidence must be presented to support the recommendations and actions chosen. Dr. Green also mentioned ongoing intervention trials to establish the effect of attained genetic risks information for common diseases, e.g., type 2 diabetes or obesity. He also mentioned the forthcoming MedSeq study, which is the first clinical trial ever funded by the National Institutes of Health $(\mathrm{NIH})$ to empirically study the use of whole genome sequencing in the practice of medicine and which is expected to meet the challenges of disclosure of largescale genomic data. Dr. Green finalized by citing a statement given by the US Preventive Services Task Force (Petitti et al. 2009): "Decision makers do not have the luxury of waiting for certain evidence. Even though evidence is insufficient, the clinician must still provide advice, patients must make choices, and policymakers must establish policies."

Martina Cornel (VU University Medical Center Amsterdam, The Netherlands) spoke about the problems facing the application of genomics in the prevention of common diseases and focused on recently published policy statements by the European Society of Human Genetics regarding direct-toconsumer genetic testing and genetic testing for common disorders. Dr. Cornel's comprehensive and informative presentation can be followed up in her article, coauthored by Carla van El and Pascal Borry, in this issue (Cornel et al. 2012).

In his keynote speech, Ron Zimmern (Foundation for Genomics and Population Health, UK) emphasized the need for, and responsibility of, scientists to address possible misleading concepts and terminology in medical genetics and to resolve the misapprehension of genomics in translational medicine, in particular with regard to the information given to stakeholders. Clarifying the differences between the different purposes for which a genetic test might be offered will lead to a substantial improvement in regulating genomic applications in medical practice and public health. In Dr. Zimmern's view, the provision of regulatory policy statements should firstly distinguish between the use of genetic tests to confirm or exclude medical diagnosis (diagnostic testing) and the use of tests in healthy persons (predictive testing) and, secondly, within predictive genetic testing, distinguish between the use of presymptomatic (deterministic) and susceptibility (probabilistic) genetic tests. Since public interest is growing out of curiosity to undergo commercially offered genetic testing, physicians should be prepared to assist consumers to interpret these results and to give advice about their potential misleading message. Dr. Zimmern emphasized the fact that misinterpretation, misconception, and wrongful anxiety on the part of consumers and patients will only be overcome through better information, rather than through prohibition. He strongly argued against a paternalistic attitude on the part of health advisers. Dr. Zimmern's précis of his talk focusing on the community genetics perspectives of the evaluation and regulation of predictive genetic testing can be read in this issue (Zimmern 2012).

Pascal Borry (University of Leuven, Belgium) addressed ethical issues related to preconceptional carrier screening offered by direct-to-consumer companies. Although carrier testing for autosomal recessive diseases in couples with a high a priori risk for having a child with a certain disease offers benefits, there are certain constraints against the implementation of carrier screening in population-wide programs. To provide a better insight into existing attitudes towards carrier screening, Dr. Borry and his colleagues Sandra Janssens and Anne de Paepe prepared a systematic review of the literature regarding healthcare professionals' attitudes towards cystic fibrosis carrier screening, which we invite you to read in this issue (Janssens et al. 2012).

Irmgard Nippert (Women's Health Research Unit, Medical School of the University of Muenster, Germany) presented some results of a collaborative research project on cancer risk communication. The project focused on current practice of 
risk communication and management of familial breast cancer in primary care in Germany, France, The Netherlands, and the United Kingdom. Using standardized questionnaires, which were randomly distributed among general practitioners, obstetricians, and gynecologists in each country, this survey assessed primary health care providers' preferred practice responsibilities as to who should undertake tasks such as ordering genetic testing, pretest risk assessment based on family history, and genetic counseling, as well as posttest delivery of results and risk communication. A significant proportion of the general practitioners in Germany and France felt themselves competent to provide genetic risk assessment and communication, whereas in the UK and the Netherlands, general practitioners were less inclined to provide these services themselves. In contrast, obstetricians and gynecologists were more inclined to share responsibility with genetic specialists. Overall, the study revealed a disconnection between general practitioners and genetic specialists. The observed tendency is that general practitioners prefer to assess and communicate genetic risks themselves and are often unaware that they may not perform adequate risk assessment and risk communication, which may be to the detriment of patients wishing to benefit from familial cancer risk information. In this issue, Dr. Nippert and her colleagues Claire JulianReynier, Hilary Harris, Gareth Evans, Christi van Asperen, Aad Tibben, and Jörg Schmidtke present a detailed report on the outcome of the survey (Nippert et al. 2013).

Anders Nordgren (Center for Applied Ethics, Linköping University, Sweden) delivered insight into current direct-toconsumer genetic testing companies' practices in promoting their test kits, which are clearly focused on the aspects of empowerment and input to identity perception ("getting control over your life and health and learn about your personal identity"). In the scientific community, it is acknowledged that this kind of information policy might lead to misinterpretation of risk (e.g., false reassurance), possibly leading to disempowerment and distortion of identity. Dr. Nordgren concluded that, with regard to the regulation of companies offering medical tests, a differentiated, two-track approach is conceivable. On the one hand, one should encourage companies to engage in self-regulation (i.e., certification and mandatory provision of genetic counseling); on the other, officially imposed national and international regulation might be appropriate for those companies not prepared to do so. Read more about this in the article by Dr. Nordgren which is published in this issue (Nordgren 2012).

Hans-Hermann Dubben (University Medical Center Hamburg-Eppendorf, Germany) discussed the question whether benefits outweigh risks of cancer-screening programs (e.g., PSA-testing for prostate cancer, mammography for breast cancer, and colonoscopy for colorectal cancer types) on the basis of currently available study data. He stated that experiences from cancer-screening trials might also apply to studies on potential benefits and risks of genetic screening. For example, prostate cancer screening programs (e.g., European Randomized Study of Screening for Prostate Cancer) have shown limited evidence so far for cancer-specific mortality reduction. On the contrary, in the course of screening, many false-positive diagnoses occurred, followed by unnecessary biopsies and psychological harm to the individuals. Moreover, there was overdiagnosis and overtreatment, i.e., unnecessary treatment of indolent cancers that would not become symptomatic or cause death. Dr. Dubben pointed out that, for statistical reasons, cancer screening studies require at least several hundred thousand participants. Another considerable drawback of the studies is that they are based on insufficient follow-up times and, additionally, on certain methodical problems or imprecisions. In fact, all studies to date (including systematic reviews) have too little power to detect relevant differences in cancer-specific mortality and thus are still inconclusive. For those reasons, accurate interpretation as to whether the beneficial effects outweigh potential harm cannot be assessed in trials, a statement that might also be true for other diseases, e.g., genetic diseases. Due to the nature of chronic diseases, results only become available decades after trial initiation. By that time, they are probably antiquated because they refer to a situation (population, lifestyle, diagnostics, treatment options) many years previously. Dr. Dubben concluded that doctors have to be well informed in order to adequately explain the pros and cons of screening programs to enable individuals to make an informed decision.

Norbert Paul (Institute of History, Philosophy, and Ethics of Medicine, Johannes Gutenberg-University Mainz, Germany) argued that health care systems are based on shared responsibility between the individual and the community. The appreciation of autonomy is fueled by a shift from public to personal responsibility for health in most Western health care systems. Against this background, an increased knowledge about individual health-related risks will —in the ideal case-lead to an increase in the ethically and socially dominant principle of autonomy. On the other hand, risk-adjusted, health-promoting behavior is reshaped into a social obligation and, in fact, sets limits to individual autonomy. Predictive genetic information, increasingly marketed as a means of empowering individuals to control their personal risk and to take charge of their biological future, reallocates emphasis onto individual responsibility, despite its commonly small predictive power and the restricted potential of controlling health risks. The public notion of genetic testing reintroduces a deterministic view of the gene and creates a novel genetic exceptionalism arising from misconception of its impact. Dr. Paul and his colleagues, Mita Banerjee and Susanne Michl, discuss these "captious certainties" in their article in this issue (Paul et al. 2013).

The second keynote talk of the symposium was given by Gerd Gigerenzer (Harding Center for Risk Literacy and Max 
Planck Institute for Human Development, Germany). Dr. Gigerenzer started with a quote "In the Western world, we have taught most citizens to read and write, but have fallen short of teaching them to understand risks." If patients and doctors do not understand risks, informed decision making is, more than ever, illusory. There is a significant lack of efficient training in risk communication in medical schools and the educational system in general. Deception often begins with the press and scientific journals. Wrong (risk) information (overstating risk and understating harm) can lead to wrong policies and unnecessary treatment interventions. Misinterpretation of statistical risks can, thus, cause harm, more than benefit. Dr. Gigerenzer illustrated the misperception of the public and of physicians, showing data from prostate (PSA) and breast cancer (mammography) screening programs. Overall, these programs have achieved little or no reduction in mortality rates from these specific cancer types, but, as Dr. Gigerenzer showed in his slides, people still believe in this potential by attending those screening programs. The conclusion Dr. Gigerenzer drew was that no information can therefore even mean "better" information- "less is more". In medical care, the communication of natural frequencies instead of conditional probabilities, of mortality rates instead of 5-years survival rates, and of absolute risks instead of relative risks, would greatly improve the implementation and effectiveness of necessary prevention strategies and also reduce psychological and, sometimes also, physical harm to patients.

Kai Insa Schneider (Hannover Medical School, Germany) reported results from a comprehensive literature review (1990-2011) on the subject of compliance among patients and unaffected persons following genetic testing. The review, which is published in this issue (Schneider and Schmidtke 2013), focuses on the following three questions: (1) Is there a difference in the compliance between persons (e.g., colon or breast cancer patients or their immediate unaffected relatives) who received a positive genetic test result as against persons who received a negative test result from genetic testing? (2) Is adherence to doctor's recommendations (e.g., intake of medication or behavioral changes concerning, for example, physical activity or diet) influenced by genetic testing? (3) Is there a difference between genetic versus non-genetic risk information with regard to their effect on patients' compliance? More than 400 publications were screened, of which 290 were taken into consideration for evaluation according to the abovementioned criteria. Individuals (patients and nonaffected relatives at elevated genetic risk) who received a HNPCC positive test result showed greater compliance with regular cancer screening compared to individuals in whom no mutation could be detected. Interestingly, also in conditions likely to be perceived as less threatening (e.g., hemochromatosis, thrombophilia, or obesity), compliance attained in persons tested as positive was considerably higher than in persons with a negative test result. Women at increased genetic risk who underwent genetic testing for BRCA1/2 mutations subjected themselves more frequently to follow-up surveillance after having received a positive test result compared to those in whom a mutation could not be detected. Risk information based on blood tests or physical examinations appeared as effective as positive genetic test results with regard to participants' intention to undertake behavioral changes. The major result is that overall compliance of patients after receiving a high-risk estimate from genetic testing for a given condition is high. However, significant behavior change does not take place just because the analyte is "genetic." Many more factors play a role in the complex process of behavioral tuning.

The last two talks presented by Cinnamon Bloss (Scripps Translational Science Institute, USA) and Andreas Baxevanis (National Human Genome Research Institute, NIH, USA) presented data from ongoing studies - the Scripps Genomic Health Initiative (in cooperation with Navigenics) and the Multiplex Initiative, respectively. Both studies assessed preand posttest individuals' attitudes with regard to the personal impact of susceptibility genetic testing for various common health conditions. The studies only included low penetrance genetic risk markers such as common single-nucleotide variants (SNVs).

Dr. Bloss's study enrolled 4,891 adults, who received a personal genomic risk assessment for 23 health conditions as well as ancestry information; of those, 2,240 completed longterm follow-up (>12 month) through web-based questionnaires. Findings showed no measurable impact on the degree of anxiety or change in lifestyle habits. Approximately one third of all follow-up participants shared the results with their physicians (recently published in Darst et al. 2013). A proportionately higher number of participants in this group acknowledged genetic testing as "very valuable" as compared to the group of those who did not share results with their physician. Privacy concerns and overall concern about genomic testing were more prevalent in non-sharers. Taken together, the study results suggest minimal impact - positive or negative - on primary disease prevention in adult individuals. Dr. Bloss finalized with an outlook on future risk assessments in younger individuals (e.g., high school students), who may be more amenable to adopting a healthy lifestyle or to giving up potentially health damaging lifestyle habits when presented with their genomic risk profile.

The Multiplex Initiative developed its own web-based survey tool and results display which differed slightly from that used by Navigenetics. Genetic risk profiles for eight health conditions based on selected common SNVs with strong replication evidence and odds ratios between 1.25 and 2.0 were mailed as printed booklets to participants and accompanying telephone interviews were conducted. However, only $13 \%$ of participants who completed the baseline survey and visited the study homepage actually took part in the genetic testing. Those individuals were characterized by a 
strong motivation to change their behavior, high genetic literacy (i.e., they understood genetic risks as probabilistic, not deterministic) and they were internet-savvy (Kaphingst et al. 2012). Most of them shared their test results with family members, very few consulted or intended to consult their primary physician, and visits to specialist doctors did not increase significantly after testing (Reid et al. 2012). Overall, those who chose to be tested did tend to see physicians more often than non-tested persons. Dr. Baxevanis emphasized that no negative effects produced by knowledge of personalized genetic risk information were observed within this study, but he acknowledged that differences in perception between different groups and individuals might exist. To overcome problems in the way genetic risk information is conveyed to, and understood by the public, adequate information is needed and evidence-based communication strategies as well as in-person support are required.

Following the speakers' session, the symposium ended with a plenary discussion, held in German, which was chaired by Thomas Wienker from the Max Planck Institute for Molecular Genetics, Berlin. Peter Dabrock (Dep. Theology, Friedrich-Alexander University Erlangen-Nürnberg), Irmgard Nippert, Marcella Rietschel (Dep. Genetic Epidemiology in Psychiatry, Central Institute of Mental Health, Mannheim), Ralf Schwarzer (Dep. Health Psychology, Freie Universität Berlin), Ludwig Siep (Faculty of Philosophy, Westfälische Wilhelms-University Münster), and Malte Spielmann (Institute of Medical Genetics and Human Genetics, Charité, Berlin) were the podium guests. The full discussion was videotaped and a shortened version can be viewed on the following website: http://www.rki.de/DE/ Content/Kommissionen/GendiagnostikKommission/ Symposium/symposium_node.html;jsessionid= 2CD43F6E8E5454507C61822BAE13FA56.2_cid390. The conclusion reached at the discussion was that most tests offered directly to consumers solely satisfy curiosity, but otherwise lack benefit (i.e., they either are of questionable or no demonstrable meaning). The preliminary evidence drawn from the results of the studies undertaken at Scripps Translational Institute and at the NIH and presented by Dr. Bloss and Dr. Baxevanis was that the potential benefit of recently available direct-to-consumer genetic tests lies in the provision of an alleged feeling of security or, as Peter Dabrock, Professor of Theology and Ethics expressed it, the tests "serve as a secular sacraments' surrogate."

It still remains unclear whether the increasing amount of information (e.g., generated by whole genome sequencing) will improve patients' clinical outcomes, risk perceptions, and/or their motivation for behavior change. However, as Ioannidis and Khoury described in their article "Improving Validation Practices in 'Omics' Research" (Ioannidis and Khoury 2011), there are numerous and challenging steps to be taken to translate "Omics" research into health care, i.e., to present solid scientific evidence to support recommendations and actions.

We would like to thank our international expert guests for giving their time and care to make this special issue possible. We would also like to thank the peer reviewers for their valuable contributions.

\section{References}

Cornel M, El C, Borry P (2012) The challenge of implementing genetic tests with clinical utility while avoiding unsound applications. J Community Genet. doi:10.1007/s12687-012-0121-1

Darst BF, Madlensky L, Schork NJ et al (2013) Characteristics of genomic test consumers who spontaneously share results with their health care provider. Health Commun. doi:10.1080/10410236. 2012.717216

Ioannidis JP, Khoury MJ (2011) Improving validation practices in "Omics" research. Science 334(6060):1230-1232

Janssens S, Paepe A, Borry P (2012) Attitudes of health care professionals toward carrier screening for cystic fibrosis. A review of the literature. J Community Genet. doi:10.1007/s12687-012-0131-z

Kaphingst KA, McBride CM, Wade C et al (2012) Patients' understanding of and responses to multiplex genetic susceptibility test results. Genet Med 14(7):681-687

Nippert I, Julian-Reynier C, Harris H, Evans G, van Asperen CJ, Tibben A, Schmidtke J (2013) Cancer risk communication, predictive testing and management in France, Germany, the Netherlands and the UK: general practitioners' and breast surgeons' current practice and preferred practice responsibilities. J Community Genet. doi:10. 1007/s12687-013-0173-x

Nordgren A (2012) Neither as harmful as feared by critics nor as empowering as promised by providers: risk information offered direct to consumer by personal genomics companies. J Community Genet. doi:10.1007/s12687-012-0094-0

Paul N, Banerjee M, Michl S (2013) Captious certainties: makings, meanings and misreadings of consumer-oriented genetic testing. $\mathrm{J}$ Community Genet. doi:10.1007/s12687-013-0172-y

Petitti DB, Teutsch SM, Barton MB et al (2009) Update on the methods of the US Preventive Services Task Force: insufficient evidence. Ann Intern Med 150(3):199-205

Reid RJ, McBride CM, Alford SH et al (2012) Association between health-service use and multiplex genetic testing. Genet Med 14(10):852-859

Schneider KI, Schmidtke J (2013) Patient compliance based on genetic medicine: a literature review. J Community Genet. doi:10.1007/ s12687-013-0160-2

Zimmern RL (2012) Issues concerning the evaluation and regulation of predictive genetic testing. J Community Genet. doi:10.1007/ s12687-012-0111-3 